\title{
Biological control of Lygus lineolaris (Hemiptera: Miridae) and Frankliniella occidentalis (Thysanoptera: Thripidae) by Bombus impatiens (Hymenoptera: Apidae) vectored Beauveria bassiana in greenhouse sweet pepper
}

\author{
Mohammad S. Al-mazra'awi a , Les Shipp ${ }^{\text {b,*, }}$ Bruce Broadbent ${ }^{\text {c }}$, Peter Kevan ${ }^{\text {d }}$ \\ ${ }^{a}$ Department of Biotechnology, Al-Balqa Applied University, Assalt 19117, Jordan \\ ${ }^{\mathrm{b}}$ Agriculture and Agri-Food Canada, Greenhouse and Processing Crop Research Centre, Harrow, Ont., Canada N0R 1G0 \\ ${ }^{\mathrm{c}}$ Agriculture and Agri-Food Canada, Southern Crop Protection and Food Research Centre, London, Ont., Canada N5V 4T3 \\ ${ }^{\mathrm{d}}$ Department of Environmental Biology, University of Guelph, Guelph, Ont., Canada N1G 2W1
}

Received 5 July 2005, and in revised form 17 October 2005; accepted 1 November 2005

Available online 18 January 2006

\begin{abstract}
The ability of bumble bees to disseminate conidia of Beauveria bassiana (Balsamo) Vuillemin from hive-mounted dispensers to greenhouse sweet peppers for the control of tarnished plant bug (TPB) and western flower thrips (WFT) was investigated in greenhouse trials using large screened enclosures. Samples collected from the enclosures (four treatments) on two sampling dates showed that 97, 90, 91, and $42 \%$ of the collected bees, flowers, leaves, and TPB, respectively, showed detectable densities of $B$. bassiana on the first sampling date. On the second sampling date, 99, 96, 87, and 30\% of collected bees, flowers, leaves, and TPB, respectively, showed detectable densities of the fungus. Mean mortalities of TPB collected from cages treated with B. bassiana were 34 and $45 \%$ compared to 9 and $15 \%$ in the controls on the first and second sampling dates, respectively. Mean infection rates of WFT were 40 and $34 \%$ compared to $3 \%$ in the controls on the first and second sampling dates, respectively. These results indicate that bumble bees are an effective means of vectoring the fungal conidia to the crop. The pollinator vector technology is a novel example of integrating agro-ecosystem processes of pollination and pest management, that reduces the reliance on insecticides and increases crop yields and quality.
\end{abstract}

(c) 2005 Elsevier Inc. All rights reserved.

Keywords: Beauveria bassiana; Bee vectoring; Bombus impatiens; Dispensers; Frankliniella occidentalis; Lygus lineolaris; Pollination

\section{Introduction}

Lygus lineolaris (Palisot de Beauvois) (Hemiptera: Miridae), the tarnished plant bug (TPB), is a serious pest for many agricultural crops and is becoming an increasingly important pest in greenhouse vegetable crops in Canada (Howard et al., 1994). The TPB is associated with weedy habitats near and inside greenhouses and the number of TPB collected from such habitats were correlated with their number inside nearby greenhouses (Gillespie et al., 2003).

\footnotetext{
* Corresponding author. Fax: +519 7382929.

E-mail address: SHIPPL@AGR.GC.CA (L.Shipp).
}

The western flower thrips (WFT), Frankliniella occidentalis (Pergande) (Thysanoptera: Thripidae) is a major pest of greenhouse sweet pepper, Capsicum annuum L. (Solanaceae) (Shipp et al., 1991, 1998). WFT causes direct yield loss by feeding on, or ovipositing in, developing fruits which results in bronzing and silvering of the fruit (Shipp et al., 1998). Although biological control measures have been adopted for management of WFT on greenhouse sweet peppers, chemical insecticides are required sometimes for effective control (Shipp et al., 1998). Insecticidal treatments, however, are the main control measures for other pests, such as the TPB, which are difficult to control with biological control agents (Shipp et al., 1998). The use of insecti- 
cides for TPB or WFT control can disrupt biological control programs for other greenhouse pests, such as aphids. In addition, the development of pesticide resistance has been reported for both WFT (Immaraju et al., 1992) and TPB (Snodgrass, 1996a,b).

The entomopathogenic fungus, Beauveria bassiana (Balsamo) Vuillemin (Hypomycetes) has been shown to be an excellent potential biological control agent for many agricultural pests (Colorado potato beetle, European corn borer, codling moth and greenhouse pests) (Goettel et al., 1990; Lewis et al., 1996; Poprawski et al., 1997). Once its infective propagules come in contact with the host, they penetrate directly through the cuticle. This route of exposure is essential for the control of pests with sucking mouthparts, which are unlikely to ingest the microbe during feeding (Dunn and Mechalas, 1963). Moreover, B. bassiana is safe for pollinators (Kevan et al., 2003) and other beneficial organisms under field conditions (Goettel et al., 1990).

Previous reports have demonstrated the susceptibility of TPB to B. bassiana (Bidochka et al., 1993; Liu et al., 2002; Steinkraus and Tugwell, 1997). The fungus causes mortality to TPB through disintegration of the insect cuticle and muscle tissues (Bidochka et al., 1993).

Beauveria bassiana has also been found to be virulent to WFT. Gindin et al. (1996) screened 16 entomopathogenic fungal isolates belonging to four species including $B$. bassiana for their virulence against WFT and onion thrips, Thrips tabaci Lindeman (Thysanoptera: Thripidae). All tested isolates caused mortalities between 58.2 and $89.5 \%$ for the WFT and 96.0 and $100 \%$ for the onion thrips (Gindin et al., 1996). Jacobson et al. (2001) tested two commercial formulations of $B$. bassiana Naturalis-L (Troy BioSciences Inc., USA) and BotaniGard WP (Mycotech, Butte, MT, USA), against WFT and the non-target biological control agent Neoseiulus (Amblyseius) cucumeris Oudemans (Acari: Phytoseiidae) on greenhouse cucumbers. Both products reduced populations of immature WFT by 87 and $75 \%$, respectively, compared to untreated control but showed no adverse effect on $N$. cucumeris (Jacobson et al., 2001). B. bassiana combined with insect attractants such as Lure Insect attractant (Setre Chemical Co., Memphis, TN, USA) and sugar was also evaluated against WFT (Ludwig and Oetting, 2002). Although the attractants did not enhance the efficacy of $B$. bassiana, high populations of WFT were reduced by the application of the fungus (Ludwig and Oetting, 2002). More recently, Shipp et al. (2003) investigated the influence of greenhouse microclimate on the efficacy of $B$. bassiana against greenhouse pests and their natural enemies including the WFT. In greenhouse trials, the commercial product BotaniGard ES (Emerald BioAgriculture Corp., Salt Lake City, UT, USA) which is based on B. bassiana strain GHA suppressed the population density of WFT compared to the untreated control (Shipp et al., 2003).

A novel method for the application of microbial control agents against agricultural pests using bee pollinators has been developed after honey bees were evaluated as vectors of fungi that were antagonistic to gray mold, Botrytis cinerea Pers. (Moniliaceae) on strawberries in Ontario (Peng et al., 1992) and the bacterium, Pseudomonas fluorescens (Trevisan) (Pseudomonadaceae), which is antagonistic to fire blight Erwinia amylovora (Burrill) (Enterobacteriaceae) in apples and pears in western USA (Thomson et al., 1992). Later, the same principle was used successfully against gray mold on raspberries using both honey bees and bumble bees as vectors (Yu and Sutton, 1997). This technology was tried in other countries when the fungus Trichoderma harzianum Rifai (Hypocreaceae), which is antagonistic to gray mold, was applied to strawberries by honey bees in Italy (Maccagnani et al., 1999) and by bumble bees and honey bees in the USA (Kovach et al., 2000).

The first report on using bee pollinators to deliver microbial control agents against insect pests was by Gross et al. (1994), who showed that honey bees effectively vectored Heliothis nuclear polyhedrosis virus to crimson clover against Helicoverpa zea (Boddie) (Lepidoptera: Tortricidae) (Gross et al., 1994). Later, the entomopathogenic fungus, Metarhizium anisopliae (Metsch.) Sorokin (Hypomycetes), was vectored successfully by honey bees to winter and spring canola for the control of the pollen beetle, Meligethes aeneus F. (Coleoptera: Nitidulidae), in the UK (Butt et al., 1998). The bee-delivered spores resulted in mortalities of 47 and $64 \%$ compared to 5 and $12 \%$ in the controls on winter and spring canola, respectively (Butt et al., 1998).

Given the virulence of B. bassiana to TPB and WFT, and the widespread use of bumble bees as pollinators for many greenhouse crops including sweet peppers (Shipp et al., 1994), bumble bees, Bombus impatiens (Hymenoptera: Apidae), were investigated as a vector for $B$. bassiana for the control of WFT and TPB on greenhouse sweet peppers.

\section{Materials and methods}

\subsection{Insect and plant cultures}

Newly emerged adults of TPB were obtained from a laboratory culture maintained in the insectary at the AAFC, Southern Crop Protection and Food Research Centre (SCPFRC), London, ON, Canada. This culture was established from field-collected TPB adults on alfalfa at the SCPFRC research farm and kept in cages containing green beans, potato sprouts and romaine lettuce at $24^{\circ} \mathrm{C}, 60 \%$ relative humidity $(\mathrm{RH})$ and $16 \mathrm{~h}$ photoperiod.

Colonies of the bumble bee, B. impatiens (BioBest Canada, Leamington, ON, Canada), were used during the experiment. Each colony consisted of a queen and 40-50 workers. The bees in each colony were transferred from their cardboard colony box to a wooden colony box $(32 \mathrm{~cm} \times 23 \mathrm{~cm} \times 24 \mathrm{~cm})$ that allowed the use of an inoculum dispenser. Bees were kept in the wooden boxes for $48 \mathrm{~h}$ before use in the trials to allow for acclimation to the new chamber. 
The inoculum dispenser was similar to that used by $\mathrm{Yu}$ and Sutton (1997) with a slight modification. The dispenser consisted of a wooden box $(21 \mathrm{~cm} \times 120 \mathrm{~cm} \times 8.5 \mathrm{~cm}$ height $)$ that was divided horizontally into two compartments by a 7-mm thick sheet of Perspex. The lower compartment had a sliding tray with a zigzag passageway in which the inoculum was spread $5 \mathrm{~mm}$ deep. Each of the lower and upper compartments were connected to the main chamber of the hive by a hole $13 \mathrm{~mm}$ in diameter. The hole connecting the upper compartment with the main chamber was supplied with a tapered tube that projected inside the main chamber allowing the bees to enter the main chamber but not to go out. This insured that all exiting bees left through the lower compartment where the formulated inoculum was placed. After crawling through the inoculum dispenser, the bees exited via a hole made in the corner of the Perspex sheet to the upper compartment where they left the hive through the hole in the front of the dispenser. Returning bees entered via the hole in the front of the upper compartment and proceeded to the hole in the main chamber of the hive without contacting the inoculum in the lower compartment. The modification to the dispenser involved replacing the front wooden side of the dispenser with a sheet of Perspex. This allowed more light to enter through the dispenser facilitating bees exiting the main chamber of the hive to the dispenser and then to the outside. Each hive was used for two successive sampling dates. Before the vectoring trials and between sampling date trials, the bees were supplied with pollen mixed with $50 \%$ wt./wt. sugar syrup.

Sweet pepper (cv Edison) plants were grown in a 1:1 mixture of sand and peat moss in $20-\mathrm{cm}$ pots. Initially, the plants were maintained in a separate greenhouse compartment until the first and second flower cluster stages and then transferred to the screened cages for the vectoring trials. Day/night temperature set points for the greenhouse were $22 / 19^{\circ} \mathrm{C}$ and $70 \%$ RH. Greenhouse climate was maintained by Argus Control Systems (Argus Control Systems, White Rock, BC, Canada). Plants were irrigated and fertilized with the Harrow Fertigation Manager (Labbate Climate Control Systems, Leamington, ON, Canada) according to standard commercial practices (Ontario Ministry of Agriculture and Food, 2001).

\subsection{Inoculum preparation}

Beauveria bassiana strain GHA (BotaniGard WP, Emerald BioAgriculture, Salt Lake City, UT, USA) was used in the vectoring trials. For each replicate, to assess the number of colony forming units (CFU) per unit weight, six $0.1 \mathrm{~g}$ samples were taken at random, suspended in $100 \mathrm{~mL}$ sterile distilled water and $0.1 \%$ Tween 80 , and then agitated on a rotary shaker at $125 \mathrm{rpm}$ for $2 \mathrm{~h}$. Three 0.1 aliquots of $10-$ fold serial dilutions of each suspension were spread on oatmeal agar Petri plates amended with $550 \mu \mathrm{g} / \mathrm{mL}$ Dodine, $400 \mu \mathrm{g} / \mathrm{mL}$ penicillin $\mathrm{G}, 1000 \mu \mathrm{g} / \mathrm{mL}$ streptomycin sulfate and $5 \mu \mathrm{g} / \mathrm{mL}$ crystal violet (Beilharz et al., 1982), incubated at $25 \pm 1^{\circ} \mathrm{C}$ for $4-5$ days, after which colonies of B. bassi- ana were counted and recorded. To determine the viability of the conidia, six $0.01 \mathrm{~g}$ samples of conidia were suspended in $100 \mathrm{~mL}$ distilled water and $0.1 \%$ Tween 80 . Then, $200 \mu \mathrm{L}$ of the conidial suspension was added to $1 \mathrm{~mL}$ of Sabouraud's dextrose broth amended with $1 \%$ yeast extract in a sterile test tube. The suspension was incubated at $24 \pm 1{ }^{\circ} \mathrm{C}$ for $24 \mathrm{~h}$. After this, four groups each consisting of 200 conidia were examined for percentage germination using a hemacytometer and a compound microscope. Inoculum from the commercial product was prepared by mixing the dry conidia with corn flour (particle size $45-90 \mu \mathrm{m}$ ) to a concentration of $1 \times 10^{9} \mathrm{CFU} / \mathrm{g}$.

\subsection{Vectoring trials}

Vectoring trials were done at the Greenhouse and Processing Crop Research Centre, Harrow, ON, Canada, in the spring of 2003. Two adjacent climate-controlled greenhouse compartments $(8.5 \mathrm{~m} \times 13 \mathrm{~m})$ were used for the trials. Two translucent screened cages $(1.8 \mathrm{~m} \times 4 \mathrm{~m} \times 2 \mathrm{~m}$ height $)$ were placed inside each greenhouse compartment. Fine mesh screening was used to contain the bees as well as the target pests on the plants. Sixty-four potted pepper plants were placed in two double rows inside each of the screened cages. The experimental design consisted of a complete randomized block design with each trial being replicated over time (block). Within each trial, all treatments were randomized among the cages. Treatments were: (1) viable B. bassiana inoculum $+\mathrm{TPB}+$ bumble bees, (2) viable $B$. bassiana inoculum + WFT + bumble bees, (3) heat-inactivated $B$. bassiana inoculum + TPB + WFT + bumble bees and (4) TPB + WFT only. Plants in three of the cages were infested with newly emerged $L$. lineolaris adults at a rate of four adults per plant (treatments 1, 3, and 4). Adults of WFT were introduced at a rate of 30 thrips per plant into three cages (treatments 2, 3, and 4). TPB and WFT adults were allowed to feed on the plants for $24 \mathrm{~h}$ and then were checked to ensure survival. Next, three bumble bee colonies in the modified wooden boxes each equipped with an inoculum dispenser were placed inside three of the cages between the two double rows of pepper plants (treatments 1,2 and 3). Two dispensers were filled each with $25 \mathrm{~g}$ of the prepared inocula (treatments 1 and 2), while the dispenser in the remaining colony was filled with $25 \mathrm{~g}$ corn flour inoculated with the heat-inactivated conidia (treatment 3 ). During the experiment in both the viable and heat-inactivated treatments, the dispensers were refilled after a 72-h interval.

After filling the dispensers, the bumble bees were allowed to forage on the flowering pepper plants. To assess dissemination of $B$. bassiana, samples were collected 3 days after the dispensers were placed on the hives. On each sampling date, 10 bumble bees, 10 fully opened pepper flowers, 10 pepper leaves, 10 TPB adults and 50 WFT adults were collected from each treated cage. Bumble bees were collected directly after they left the hive through the dispenser. Flowers and leaves immediately adjacent to the bee colony were not selected in the sampling process. To estimate via- 
ble conidia (CFU) on the bumble bees, flowers, leaves, TPB and WFT, samples were agitated individually in flasks containing $100 \mathrm{~mL}$ sterilized distilled water and $0.1 \%$ Tween 80 on a rotary shaker for $2 \mathrm{~h}$ except for the WFT samples that were vortexed in $1-\mathrm{mL}$ centrifuge tubes. Three 0.1 aliquots of 10-fold serial dilutions of each suspension were spread on oatmeal agar Petri plates that were prepared as described in Section 2.2. To assess mortality of TPB, adults were collected from cages dedicated to treatments 1,3 , and 4. Five subsamples, each containing 10 TPB, were collected per treatment by means of an aspirator and plastic vials. Each subsample was placed in a ventilated PVC cylinder $(10 \mathrm{~cm}$ diameter $\times 20 \mathrm{~cm}$ height $)$ lined with moist filter paper and incubated at $24^{\circ} \mathrm{C}$ and a 16 -h photoperiod. Fresh organically produced lettuce leaves were provided as food for the caged insects. Mortality was recorded on days 1, 4, and 7 after collection. Dead insects were removed and placed in Petri plates lined with moist filter paper to encourage external growth of conidia. To assess the infection rate in WFT, adults were collected from cages dedicated to treatments 2, 3, and 4. Five subsamples, each containing 10 WFT adults, were collected per treatment using an aspirator and plastic vials. The subsamples were surface sterilized by immersing in $70 \%$ ethanol for $15-20 \mathrm{~s}$, followed by $5 \%$ bleach containing $0.3 \% \mathrm{NaOCl}$ amended with $0.05 \%$ Tween 20 for $3 \mathrm{~min}$ and two rinses of sterile distilled water amended with Tween 20 for $1-2 \mathrm{~min}$. The surface-sterilized WFT adults were then placed in water agar plates and incubated at $25^{\circ} \mathrm{C}$ for 5-6 days. After the incubation period, all test individuals were examined using a dissecting microscope for the presence of B. bassiana.

The experiment was replicated five times. Each replicate consisted of a new set of plants, freshly prepared inocula and new batches of insects. Between replications, the greenhouse compartments were heated to $35-37^{\circ} \mathrm{C}$ for $72 \mathrm{~h}$ and fumigated with the insecticide Nicotine (MGS Horticultural, Leamington, ON, Canada) to eliminate any carryover of TPB and WFT between replicates. Mean $( \pm$ SE) daily temperature and $\mathrm{RH}$ in the cages were maintained at $21.0 \pm 0.1^{\circ} \mathrm{C}$ and $67.2 \pm 5.1 \% \mathrm{RH}$ using the Argus Greenhouse Management System (Argus Control System) to ensure similar conditions between the two greenhouse compartments. Temperature and $\mathrm{RH}$ were monitored inside the cages using shaded temperature/humidity probes (Hycal,
Elmonte, CA, USA). Probes were installed ca. $60 \mathrm{~cm}$ above the ground at the middle of the plant canopy.

\subsection{Statistical analysis}

Percentage mortalities of TPB adults and infection rates of WFT adults were arcsin square root transformed and analyzed using a mixed linear model in a split plot design with sampling dates (the subplots) and treatments (the whole plot) (PROC MIXED, SAS Institute, 1999). Means were then separated using polynomial contrasts where the mortality and infection rates in the Beauveria-treated cages were compared to the average mortality from both the heat-inactivated Beauveria and non-Beauveria treatments. Another contrast was made to compare the effects of the heat-inactivated Beauveria and the non-Beauveria treatments. Data on the estimated values of B. bassiana CFU were $\log (x+1)$ transformed and subjected to ANOVA to test for differences in CFU counts between the two sampling dates (PROC ANOVA, SAS Institute, 1999). For both CFU data and mortality and infection rate data, means were compared only if significant differences were found by the $F$-test. The type I error rate $(\alpha)$ was set at 0.05 level for all the tests.

\section{Results}

Bumble bees leaving the hive on the two sampling dates efficiently acquired $B$. bassiana conidia supplied via the hive dispensers. Essentially, all the bee samples had detectable levels of the fungus (Table 1). Inocula carried by the emerging bees ranged from 0 to $2.1 \times 10^{6}$ and 0 to $2.3 \times 10^{6} \mathrm{CFU} /$ bee on the first and second sampling dates, respectively. The bumble bees were also effective in delivering the inoculum to the flowers of greenhouse sweet peppers. Ninety percent $( \pm 5.2)$ of the flowers on the first sampling date and $95.6 \%( \pm 2.4)$ on the second sampling date contained detectable densities of the fungus (Table 1). Densities of CFU per flower were from 0 to $1.4 \times 10^{5}$ and 0 to $2.1 \times 10^{5}$ on the first and second sampling dates, respectively. Inoculum of B. bassiana was also detected on the leaves of the crop. Percentages of the leaf samples with detected densities of the fungus were $91.3 \%( \pm 3.0)$ and $86.7 \%( \pm 4.4)$ on the first and second sampling dates, respectively (Table 1). Densities of

Table 1

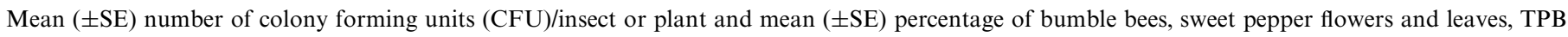
adults and WFT adults with detectable densities of Beauveria bassiana as collected from treatments containing bumble bees and B. bassiana

\begin{tabular}{|c|c|c|c|c|}
\hline \multirow[t]{2}{*}{ Sample } & \multicolumn{2}{|l|}{ First sampling date } & \multicolumn{2}{|l|}{ Second sampling date } \\
\hline & Mean & Percentage & Mean & Percentage \\
\hline Bees & $4.5 \times 10^{5}\left(5.3 \times 10^{4}\right)$ & $97.0(1.5)$ & $3.8 \times 10^{5}\left(7.9 \times 10^{4}\right)$ & $98.9(1.1)$ \\
\hline Flowers & $1.8 \times 10^{4}\left(4.7 \times 10^{3}\right)$ & $90.0(5.2)$ & $3.2 \times 10^{4}\left(9.0 \times 10^{3}\right)$ & $95.6(2.4)$ \\
\hline Leaves & $3.2 \times 10^{4}\left(7.9 \times 10^{3}\right)$ & $91.3(3.0)$ & $3.6 \times 10^{4}\left(1.4 \times 10^{4}\right)$ & $86.7(4.4)$ \\
\hline TPB & $586.7(252.7)$ & $42.0(12.4)$ & $708.3(461.6)$ & $30.0(14.7)$ \\
\hline WFT & $1013.5(502.1)$ & NA & $1031.6(285.5)$ & NA \\
\hline
\end{tabular}

CFU for WFT were based on groups of 10 WFT per group. 
the fungus were 0 to $1.7 \times 10^{5}$ and 0 to $2.0 \times 10^{5} \mathrm{CFU} /$ leaf on the first and second sampling dates, respectively. With respect to TPB adults, ca. $30-40 \%$ of the bugs showed detectable amounts of the fungus on the two sampling dates (Table 1). Densities of CFU per adult TPB were from 0 to $5.5 \times 10^{3}$ on the first sampling date and 0 to $8.2 \times 10^{3}$ on the second sampling date. Adult WFT also acquired the bee-delivered inocula with densities of CFU per subsample of 10 WFT adults ranging between 133 and 2267 and 180 and 2246 on the first and second sampling dates, respectively. No significant differences were found in the density of $B$. bassiana conidia on bumble bees $\left(F_{1,17}=0.1, P=0.75\right)$, leaves $\left(F_{1,15}=0.2, P=0.66\right)$, flowers $\left(F_{1,17}=1.53, P=0.23\right)$, TPB $\left(F_{1,7}=0.23, P=0.65\right)$ and WFT $\left(F_{1,6}=0.44, P=0.53\right)$ between the first and the second sampling dates (Table 1).

Percentage mortalities of TPB collected on the two sampling dates from the cages with or without $B$. bassiana delivered by bumble bees are presented in Table 2 . Statistical analysis showed that the effects of sampling dates $\left(F_{1,9}=2.92, P=0.12\right)$ and the interaction between sampling dates and treatments $\left(F_{2,9}=3.42, P=0.08\right)$ on TPB mortality were not significant. However, significant differences in TPB mortalities were found among the treatments $\left(F_{2,8}=37.85, P<0.001\right)$. Further means testing of TPB mortality in the Beauveria-treated cages with those from the heat-inactivated Beauveria and non-Beauveria treatments, and TPB mortality in the heat-inactivated Beauveria to non-Beauveria treatments revealed that adult TPB collected from the treatments receiving the living conidia of $B$. bassiana had significantly higher mortalities than did TPB collected from cages with the heat-inactivated or non- $B$. bassiana treatments $\left(F_{1,8}=75.59, P<0.001\right)$. No significant difference in mortality of TPB was found between the heatinactivated Beauveria and the non-Beauveria treatments $\left(F_{1,8}=0.11, P=0.75\right)$. Most of the mortality occurred 3-7 days after collection of the TPB and the final cumulative mortalities 7 days after collection of the TPB ranged from 34 to $45 \%$ over the two sampling dates (Table 2).

The higher mortality of TPB collected from the living Beauveria treatment compared to the heat-inactivated Beauveria and non-Beauveria treatments was confirmed by the high percentage of dead TPB that developed external mycosis. The mean percentage of mycosed insects was $90 \%$ $( \pm 3.1)$ and $91 \%( \pm 3.0)$ for the first and second sampling dates, respectively. Few mycosed insects were observed in the heat-inactivated Beauveria and non-Beauveria treat- ments from the first and the second sampling dates (Table 2).

Similar results were obtained from analysis of the WFT infection rates (Table 3). The effect of sampling dates and the interaction effect between treatments and sampling dates were not significant at the 0.05 level $\left(F_{1,12}=1.11\right.$, $P=0.31$ for sampling date and $F_{2,12}=0.15, P=0.86$ for the interaction effect). The infection rate for the viable Beauveria treatment was significantly higher than the average values from the heat-inactivated Beauveria and the nonBeauveria treatments $\left(F_{1,12}=33.89, P<0.001\right)$ (Table 3). No significant differences were detected by comparing WFT mortalities for the heat-inactivated Beauveria treatment with non-Beauveria treatment $\left(F_{1,12}=0.2, P=0.67\right)$.

The frequency distribution of bumble bees with detectable densities of Beauveria collected from the cages supplied with the living B. bassiana is presented in Fig. 1. Only $2 \%$ of the bumble bees left the hive without detectable densities of the fungus and almost one-third of the bees carried between 1 and $1 \times 10^{5} \mathrm{CFU} / \mathrm{bee}$. The percentage of bees with Beauveria decreased with an increase in the density of CFU per bee above $10^{5}$ and $9 \%$ of the bumble bees carried more than $10^{6} \mathrm{CFU} /$ bee. Mean CFU per bee for all sampled bees was $4.2 \times 10^{5}\left( \pm 6.6 \times 10^{4}\right)$ and the median was $2.5 \times 10^{5}$ (Fig. 1).

The frequency distribution of greenhouse sweet pepper flowers sampled on the first sampling date showed that $73 \%$ of the flowers had CFU densities within the ranges 1 to $10 \times 10^{3}(34 \%), 10.1 \times 10^{3}$ to $20 \times 10^{3}(26 \%)$ and $30.1 \times 10^{3}$ to $40 \times 10^{3}(13 \%) \mathrm{CFU} /$ flower (Fig. 2). Ten percentage of the sampled flowers had no detected densities of the fungus. Few flowers had inoculum densities within the ranges of

Table 3

Mean infection rate $( \pm \mathrm{SE})$ of WFT adults on greenhouse sweet pepper plants caged without bumble bees, with bumble bees and with bumble bees and $B$. bassiana for two sampling dates

\begin{tabular}{lll}
\hline Treatment & \multicolumn{2}{l}{ Infection rate } \\
\cline { 2 - 3 } & $\begin{array}{ll}\text { First sampling } \\
\text { date }\end{array}$ & $\begin{array}{l}\text { Second sampling } \\
\text { date }\end{array}$ \\
\hline WFT + bees + B. bassiana & $39.5 \pm 11.8 \mathrm{a}$ & $34.1 \pm 6.9 \mathrm{a}$ \\
WFT + bees + heat-inactivated & $3.4 \pm 2.6 \mathrm{~b}$ & $3.1 \pm 2.6 \mathrm{~b}$ \\
$\begin{array}{l}\text { B. bassiana } \\
\text { WFT only }\end{array}$ & $2.2 \pm 2.2 \mathrm{~b}$ & $0.5 \pm 0.5 \mathrm{~b}$ \\
\hline
\end{tabular}

Means within columns and rows with different letters are significantly different at 0.05 level using polynomial contrast.

Table 2

Mean percentage $( \pm \mathrm{SE})$ mortality and mycosis of TPB adults on greenhouse sweet pepper plants caged without bumble bees, with bumble bees and Beauveria bassiana for two sampling dates

\begin{tabular}{|c|c|c|c|c|}
\hline \multirow[t]{2}{*}{ Treatments } & \multicolumn{2}{|c|}{ First sampling date } & \multicolumn{2}{|c|}{ Second sampling date } \\
\hline & $\%$ Mortality & $\%$ Mycosed & $\%$ Mortality & $\%$ Mycosed \\
\hline $\mathrm{TPB}+$ bees $+B$. bassiana & $33.6 \pm 6.6 \mathrm{a}$ & $90.0 \pm 3.1$ & $45.0 \pm 3.9 \mathrm{a}$ & $91.0 \pm 3.0$ \\
\hline TPB + bees + heat-inactivated $B$. bassiana & $9.2 \pm 2.7 \mathrm{~b}$ & $6.0 \pm 4.4$ & $15.3 \pm 3.2 \mathrm{~b}$ & $14.5 \pm 7.5$ \\
\hline TPB only & $14.8 \pm 4.1 \mathrm{~b}$ & 0 & $9.0 \pm 1.9 \mathrm{~b}$ & $1.7 \pm 1.7$ \\
\hline
\end{tabular}

Means within columns and rows with different letters are significantly different at 0.05 level using polynomial contrast. 


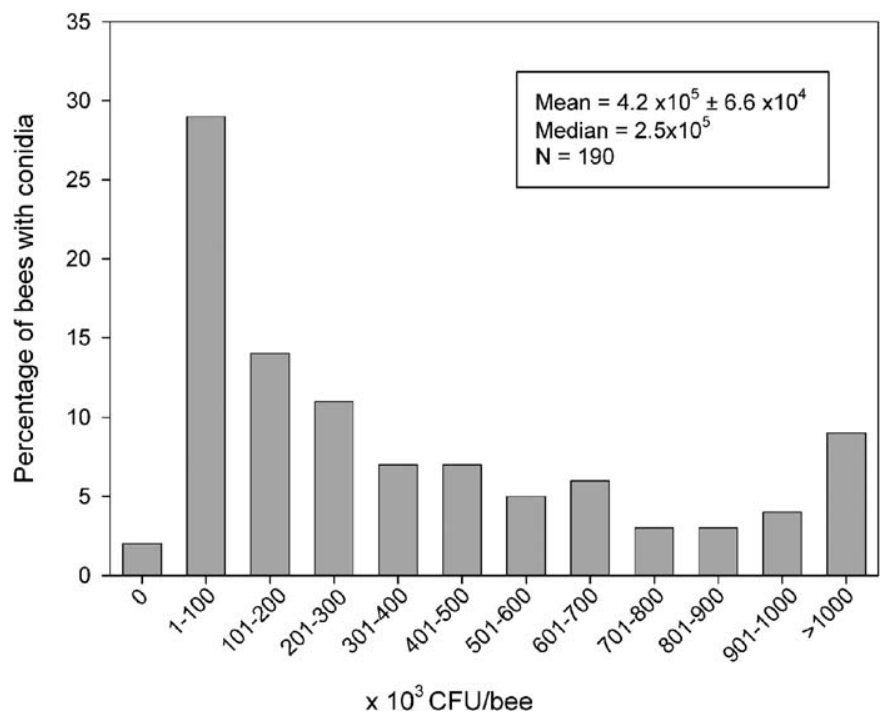

Fig. 1. Frequency distribution of collected bumble bees with detected densities of conidia of Beauveria bassiana after emerging from hive-mounted dispensers filled with $1 \times 10^{9} \mathrm{CFU}$ of $B$. bassiana per gram of corn flour.

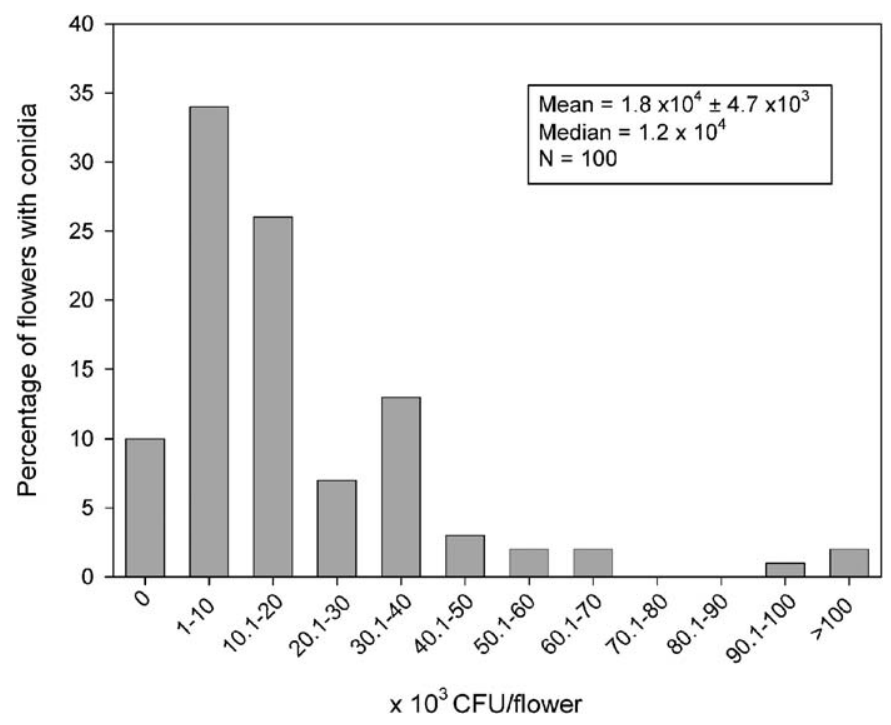

Fig. 2. Frequency distribution of sampled sweet pepper flowers with detected densities of conidia of Beauveria bassiana from the cages containing bumble bees and B. bassiana on the first sampling date.

$4 \times 10^{4}$ to $\left(1 \times 10^{5}\right.$. Mean CFU per flower for all sampled flowers on the first sampling date was $1.8 \times 10^{4}\left( \pm 4.7 \times 10^{3}\right)$ and the median was $1.2 \times 10^{4}$ (Fig. 2). On the second sampling date (Fig. 3), a small percentage (4\%) of the sampled flowers did not have any $B$. bassiana. The frequency of flowers with CFU per flower between 1 and $1.0 \times 10^{4}$, and between $1.0 \times 10^{4}$ and $2.0 \times 10^{4}$ was 31 and $29 \%$, respectively. Few flowers with inoculum densities within the range of $4.0 \times 10^{4}$ to $\left(1.0 \times 10^{5}\right.$ were sampled from the cages. Mean CFU per flower for all sampled flowers on the second sampling date was $3.2 \times 10^{4}\left( \pm 9.0 \times 10^{3}\right)$ and the median was $1.9 \times 10^{4}$ (Fig. 3).

The frequency distribution of greenhouse sweet pepper leaves (Fig. 4) with detected inocula of B. bassiana pooled

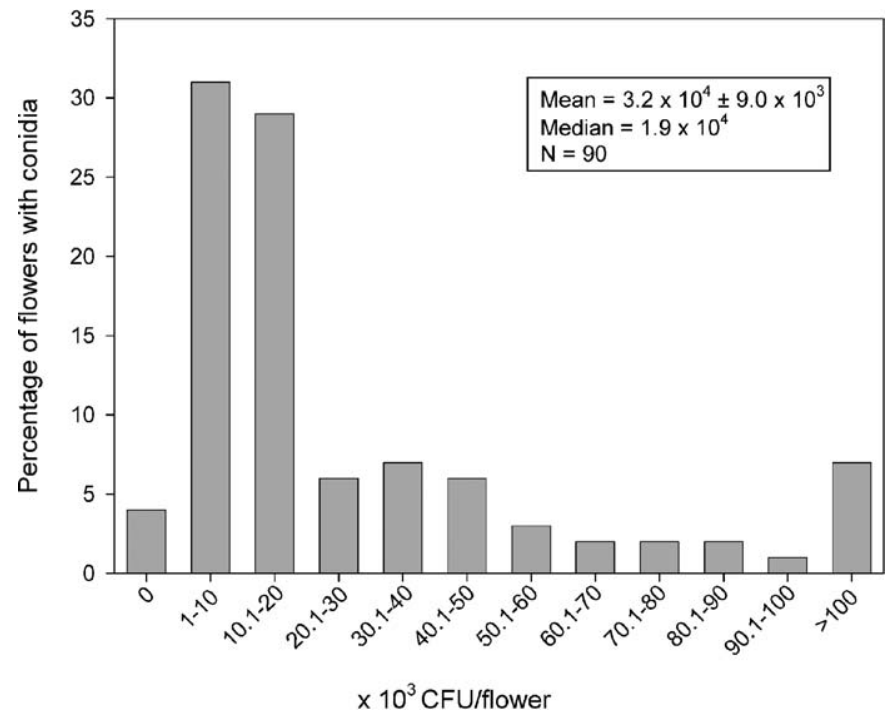

Fig. 3. Frequency distribution of sampled sweet pepper flowers with detected densities of conidia of Beauveria bassiana from the cages containing bumble bees and B. bassiana on the second sampling date.

over the sampling dates showed that $11 \%$ of the sampled leaves had no detectable $B$. bassiana. The percentage of leaves with Beauveria decreased with increasing density of CFU per leaf. The mean CFU per leaf for all sampled leaves was $3.4 \times 10^{4}\left( \pm 1.1 \times 10^{4}\right)$ and the median was $1.3 \times 10^{4}$ (Fig. 4).

A large percentage of TPB (64\%) showed no detected amounts of the fungus. The percentage of TPB with CFU densities from 1 to $1 \times 10^{3}$ and $1 \times 10^{3}$ to $2 \times 10^{3}$ were 20 and $8 \%$, respectively. Mean CFU per TPB for all sampled TPB adults was $6.5 \times 10^{2}\left( \pm 3.6 \times 10^{2}\right)$ and the median was 0 (Fig. 5).

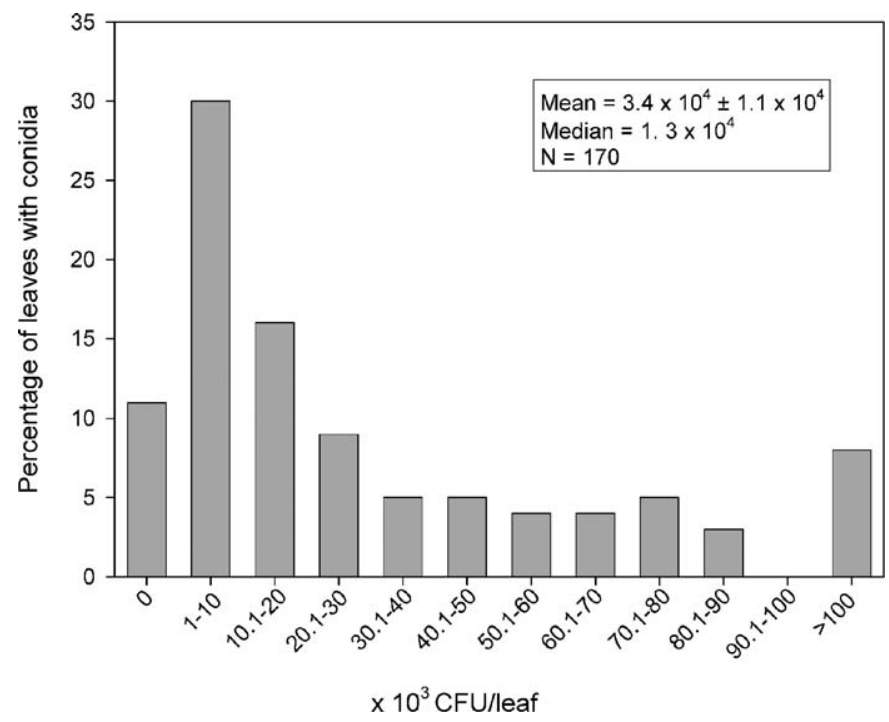

Fig. 4. Frequency distribution of sampled sweet pepper leaves with detected densities of conidia of Beauveria bassiana from cages containing bumble bees and B. bassiana pooled over the two sampling dates. 


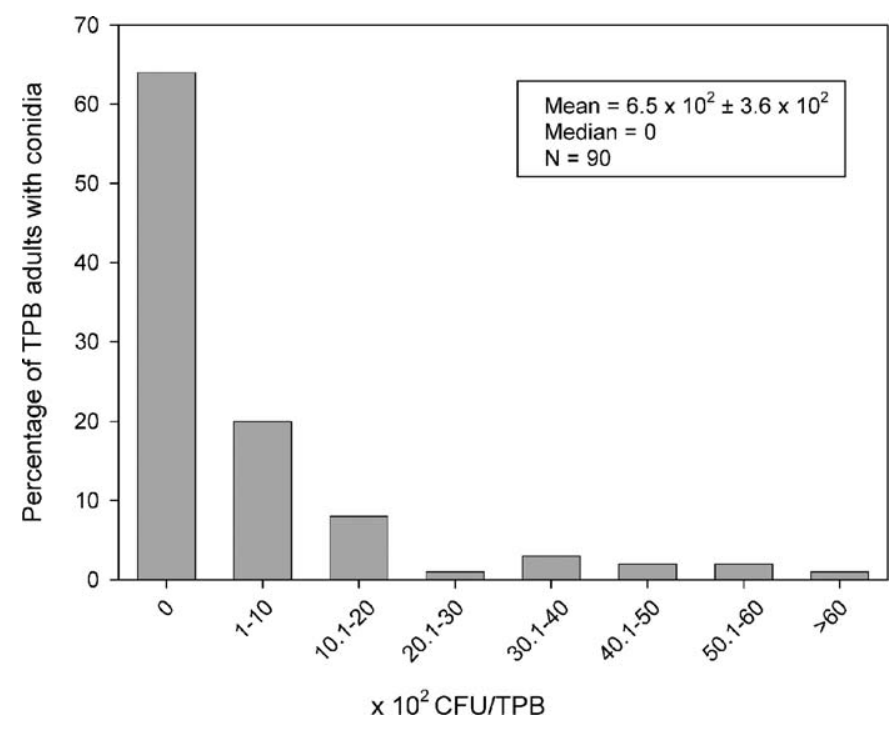

Fig. 5. Frequency distribution of collected TPB adults with detected densities of conidia of Beauveria bassiana from the cages containing bumble bees and $B$. bassiana pooled over the two sampling dates.

\section{Discussion}

This study provides the first report of using bumble bees to vector a microbial fungal biological control agent for insect control on a greenhouse crop. B. impatiens effectively vectored $B$. bassiana from the inoculum dispensers to greenhouse sweet pepper plants and subsequently to the target pests. Bumble bees in the collected samples were coated with hundreds and thousands of $B$. bassiana conidia as they left their hives. The pooled mean of $4.2 \times 10^{5} \mathrm{CFU} /$ bee for the two sampling dates was equivalent to $0.42 \mathrm{mg}$ inoculum/bee. Yu and Sutton (1997) estimated that bumble bees carried $0.4 \mathrm{mg}$ of Clonostachys (Gliocladium) roseum Bainier (Hypomycetes) inoculum when they emerged from similar dispensers.

The high percentage of flowers $((90 \%)$ on which B. bassiana was detected (Table 1) on both sampling dates indicated that the bumble bees efficiently vectored the control agent. Similar results were found by Yu and Sutton (1997), who found that $C$. rosea was recovered from $84 \%$ of the raspberry flowers treated with inoculum vectored by bumble bees. Bumble bees carried much larger amounts than they deposited on the flowers. Mean CFU per flower in the first and second sampling dates of $1.8 \times 10^{4}$ and $3.2 \times 10^{4}$, respectively, indicated that the amount detected on flowers constituted only 4.0 and $8.4 \%$ of the amount carried by the bees. Similarly, Peng et al. (1992) reported that one-tenth of the inoculum was detected on the flowers compared to the density detected on the bees when honey bees were used as vectors of $C$. rosea to strawberry for suppression of gray mold.

The density of inoculum deposited on flowers was variable and ranged from 0 to $2.1 \times 10^{5} \mathrm{CFU} /$ flower (Figs. 2 and 3). This variability might be attributed to several fac- tors including: the variable amounts of inocula carried by the bees, bee vectoring dynamics, orientation of flowers and redistribution of inocula by the bees, other organisms and air currents. Successive visitation to flowers during a foraging trip might result in a decline of the dose carried by the foraging bees and thus, would affect inoculum transfer for subsequently visited flowers. Moreover, repeated visitation by more than one foraging bee to the same flower might result in more inocula deposited on that particular flower compared to the less visited ones. This variability is common in bee delivery systems and was reported by previous investigators (Peng et al., 1992; Yu and Sutton, 1997). Mathematical models of pollen transfer (e.g., exponential decay), which might resemble conidial transfer, are frequently used to study patterns of spatial dispersal of pollen among the flowers of crops (Iwasa et al., 1995). Those models are not applicable to the current results because flower sampling in this study was done randomly, whereas collection of data for the pollen models requires sequential sampling of flowers from the pollen source (Iwasa et al., 1995; Cresswell et al., 1995). Moreover, some factors included in the model such as the total number of flowers on the plants, the proportion of visited flowers and the number of visits for individual flowers were not considered in this study.

Beauveria bassiana was also disseminated from the inoculum dispensers to the leaves of the crop. The pooled average of the two sampling dates of $3.4 \times 10^{4}$ constituted $8.0 \%$ of the amount carried by the bees (Fig. 4). The presence of the inoculum on the leaves might be attributed to sedimentation of conidia from flying bumble bees as well as to redistribution of conidia by the bees, air currents and other living organisms. Observations of bumble bee behavior during the experiment showed that some bees flew directly from the dispensers to the leaves where they groomed.

The bee-delivered B. bassiana was acquired by the TPB feeding on the crop. This was indicated by the percentages of sampled TPB with detected densities of the fungus (Fig. 5). The amount of conidia detected on the TPB adults, as expressed by the pooled mean of the two sampling dates (Fig. 5), constituted 3.6 and $2.0 \%$ of the amount detected on the flowers in the first and second sampling dates, respectively. Although these percentages are low, substantial mortality of TPB was observed in the cages treated with $B$. bassiana compared to the untreated ones (Table 2). The fungus was considered as the cause of the death of TPB adults because an high percentage of insect cadavers developed external mycosis (Table 2). Low mortalities were observed in samples collected from the heat-inactivated Beauveria or the non-Beauveria treatments which indicated that neither the carrier, fine corn flour, nor the bumble bees affected the survival of the bugs and their susceptibility to the entomopathogen.

Similar to the results of TPB mortality, the infection rates of WFT adults collected from the living Beauveria treatment were higher compared to those of WFT adults collected from the heat-inactivated Beauveria or the nonBeauveria treatments (Table 3). This was further empha- 
sized by the detection of bee-delivered conidia on WFT adults collected from cages supplied with living Beauveria. The pooled mean of the two sampling dates of $1023 \mathrm{CFU} /$ 10 WFT constituted 5.7 and $3.2 \%$ of the amount detected on the flowers on the first and second sampling dates, respectively. Very low infection rates $(0.5-3.4 \%)$ were recorded from WFT adults collected from the heat-inactivated Beauveria or the non-Beauveria treatments. The infection is likely attributed to contamination by personnel while conducting the experiment.

Results indicated that the density of $B$. bassiana propagules on bumble bees, pepper flowers and leaves, TPB and WFT did not differ between the two sampling dates. Differences on the target pests might be detected after longer periods of bee vectoring with continued filling of the dispensers to cope with depletion of the inocula.

Very little is known about the impact of B. bassiana on bumble bees. The bumble bees, B. pratorum (L.) and B. terrestris (L.), have been reported as hosts for Beauveria (Goettel et al., 1990). Although B. bassiana infected some bumble bee workers during the course of the study, no epizootic of Beauveria was observed in the colonies. Careful monitoring during and after the trials showed no adverse impact on colony health, brood production and activity of bumble bees. However, studies are warranted on the effects of B. bassiana on bumble bees when applied as a dry formulation.

This study demonstrated the potential of using bumble bees as a novel method for the application of B. bassiana to greenhouse sweet pepper for the control of TPB and WFT. More research is needed to determine the optimum concentration of B. bassiana for maximum infection levels in TPB and WFT and how often the dispensers need to be refilled. In addition, using bumble bees for the pollination of greenhouse sweet pepper has resulted in a positive impact on fruit quality and yield (Shipp et al., 1994). Other researchers have found B. bassiana to be effective against several insect pests including Lygus spp. (Bidochka et al., 1993; Liu et al., 2002; Noma and Strickler, 1999; Steinkraus and Tugwell, 1997) and WFT (Gindin et al., 1996; Jacobson et al., 2001; Shipp et al., 2003). By using bumble bees as a means of delivering $B$. bassiana, one not only reduces pest populations at a single point in time, but also has a continuous impact on the pest populations over time because bees are disseminating the control agent on a daily basis. This continuous impact is critical in greenhouse systems where conditions are favorable for pest reproduction. Using bee pollinators as the means for the delivery and dissemination of microbial control agents on greenhouse crops is a novel way of integrating agricultural practices (i.e., pollination and pest control) that will result in better quality and quantity of crops.

\section{Acknowledgments}

We thank Emerald BioAgriculture (Salt Lake City, UT, USA) for providing B. bassiana. The study was funded by
Al-Balqa Applied University, Jordan and Natural Sciences and Engineering Research Council/Biocontrol Network, Canada. For laboratory and field assistance, we thank P. Kelly, G. Wilson, S. Nathan, M. Adjaloo, K. Wang and K. Wharram.

\section{References}

Beilharz, V.C., Parbery, D.G., Swart, H.J., 1982. Dodine: a selective agent for certain soil fungi. T. Brit. Mycol. Soc. 79, 507-511.

Bidochka, M.J., Miranpuri, G.S., Khachatourians, G.G., 1993. Pathogenicity of Beauveria bassiana (Balsamo) Vuillemin toward lygus bug (Hem., Miridae). J. Appl. Entomol. 115, 313-317.

Butt, T.M., Carreck, N.L., Ibrahim, L., Williams, I.H., 1998. Honey-beemediated infection of pollen beetle (Meligethes aeneus Fab.) by the insect-pathogenic fungus, Metarhizium anisopliae. Biocontrol Sci. Technol. 8, 533-538.

Cresswell, J.E., Bassom, A.P., Bell, S.A., Collins, S.J., Kelly, T.B., 1995. Predicted pollen dispersal by honey-bees and three species of bumble-bees foraging on oil-seed rape: a comparison of three models. Funct. Ecol. 9, 829-841.

Dunn, P.H., Mechalas, B.J., 1963. The potential of Beauveria bassiana (Balsamo) Vuillemin as a microbial insecticide. J. Insect Pathol. 5, 451-459.

Gillespie, D.R., Foottit, R.G., Shipp, J.L., Schwartz, M.D., Quiring, D.M.J., Wang, K., 2003. Diversity, distribution and phenology of Lygus species (Hemiptera: Miridae) in relation to vegetable greenhouses in the lower Fraser Valley, British Columbia, and southwestern Ontario. J. Entomol. Soc. B. C. 100, 43-54.

Gindin, G., Barash, I., Raccah, B., Singer, S., Ben-Ze'ev, I.S., Klein, M., 1996. The potential of some entomopathogenic fungi as biocontrol agents against the onion thrips, Thrips tabaci and the western flower thrips, Frankliniella occidentalis. Folia Entomol. Hung. 57 (Suppl.), 37-42.

Goettel, M.S., Poprawski, T.J., Vandenberg, J.D., Li, Z., Roberts, D.W., 1990. Safety to nontarget invertebrates of fungal biocontrol agents. In: Laird, M., Lacey, L.A., Davidson, E.W. (Eds.), Safety of Microbial Insecticides. CRC Press, Boca Raton, FL, pp. 209-229.

Gross, H.R., Hamm, J.J., Carpenter, J.E., 1994. Design and application of a hive-mounted device that uses honey bees (Hymenoptera: Apidae) to disseminate Heliothis nuclear polyhedrosis virus. Environ. Entomol. 23, 492-501.

Howard, R.J., Garland, J., Seaman, W.L., 1994. Diseases and Insect Pests of Vegetable Crops in Canada. The Canadian Phytopathological Society and the Entomological Society of Canada, Ottawa, Ontario.

Immaraju, J.A., Paines, T.D., Bethke, J.A., Robb, K.L., Newman, J.P., 1992. Western flower thrips (Thysanoptera: Thripidae) resistance to insecticides in coastal California greenhouses. J. Econ. Entomol. 85, 9-14.

Iwasa, Y., de Jong, T.J., Klinkhammer, G.L., 1995. Why pollinators visit only a fraction of the open flowers on a plant: the plant's point of view. J. Evol. Biol. 8, 439-453.

Jacobson, R.J., Chandler, D., Fenlon, J., Russell, K.M., 2001. Compatibility of Beauveria bassiana (Balsamo) Vuillemin with Amblyseius cucumeris Oudemans (Acarina: Phytosiidae) to control Frankliniella occidentalis Pergande (Thysanoptera: Thripidae) on cucumber plants. Biocontrol Sci. Technol. 11, 391-400.

Kevan, P.G., Al-mazra'awi, M.S., Sutton, J.C., Tam, L., Boland, G., Broadbent, B., Thomson, S.V., Brewer, G.J., 2003. Using pollinators to deliver biological control agents against crop pests. In: Downer, R.A., Mueninghoff, J.C., Volgas, G.C. (Eds.), Pesticide Formulations and Delivery Systems: Meeting the Challenges of the Current Crop Protection Industry. American Society for Testing and Materials International, West Conshohocken, PA. ASTM STP 1430.

Kovach, J., Petzoldt, R., Harman, G.E., 2000. Use of honey bees and bumble bees to disseminate Trichoderma harzianum to strawberries for Botrytis control. Biol. Control 18, 235-242.

Lewis, L., Berry, E.C., Obrycki, J.J., Bing, L.A., 1996. Aptness of insecticides (Bacillus thuringiensis and carbofuran) with the endophytic Beauveria bassiana, in suppressing larval populations of the European corn borer. Agric. Ecosyst. Environ. 57, 27-34. 
Liu, H., Skinner, M., Parker, B.L., Brownbridge, M., 2002. Pathogenicity of Beauveria bassiana, Metarhizium anisopliae (Deuteromycotina: Hyphomycetes), and other entomopathogenic fungi against Lygus lineolaris (Hemiptera: Miridae). J. Econ. Entomol. 95, 675-681.

Ludwig, S.W., Oetting, R.D., 2002. Efficacy of Beauveria bassiana plus attractants for enhanced control of Frankliniella occidentalis (Thysanoptera: Thripidae). Fla. Entomol. 85, 270-272.

Maccagnani, B., Mocioni, M., Gullino, M.L., Ladurner, E., 1999. Application of Trichoderma harzianum by using Apis mellifera for the control of gray mold of strawberries: first results. IOBC Bull. 22, 161-164.

Noma, T., Strickler, K., 1999. Factors affecting Beauveria bassiana for control of Lygus bug (Hemiptera: Miridae) in alfalfa seed fields. J. Agric. Urban Entomol. 16, 215-233.

Ontario Ministry of Agriculture and Food, 2001. Growing Greenhouse Vegetables. Ontario Ministry of Agriculture and Food, Publication Number 371. Queen's Printer, Toronto, ON, Canada.

Peng, G., Sutton, J.C., Kevan, P.G., 1992. Effectiveness of honeybees for applying the biocontrol agent Gliocladium roseum to strawberry flowers to suppress Botrytis cinerea. Can. J. Plant Pathol. 14, 117-129.

Poprawski, T.J., Carruthers, R.I., Speese III, J., Vacek, C., Wendel, L., 1997. Early-season applications of the fungus Beauveria bassiana and introduction of the hemipteran predator, Perillus bioculatus for control of Colorado potato beetle. Biol. Control 10, 48-57.

Shipp, J.L., Boland, G.J., Shaw, L.A., 1991. Integrated pest management of disease and arthropod pets of greenhouse vegetable crops in Ontario: current status and future possibilities. Can. J. Plant Sci. 71, 887-914.
Shipp, J.L., Whitfield, G.H., Papadopoulos, A.P., 1994. Effectiveness of the bumble bee, Bombus impatiens Cr. (Hymenoptera: Apidae), as a pollinator for greenhouse sweet pepper. Sci. Hortic. 57, 29-39.

Shipp, J.L., Binns, M.R., Hao, X., Wang, K., 1998. Economic injury levels for western flower thrips (Thysanoptera: Thripidae) on greenhouse sweet pepper. J. Econ. Entomol. 91, 671-677.

Shipp, J.L., Zhang, Y., Hunt, D.W.A., Ferguson, G., 2003. Influence of humidity and greenhouse microclimate on the efficacy of Beauveria bassiana (Balsamo) for control of greenhouse arthropod pests. Environ. Entomol. 32, 1154-1163.

Snodgrass, G.L., 1996a. Insecticide resistance in field population of tarnished plant bug (Heteroptera: Miridae) in cotton in the Mississippi Delta. J. Econ. Entomol. 89, 783-790.

Snodgrass, G.L., 1996b. Glass-vial bioassay to estimate insecticide resistance in adult tarnished plant bug (Heteroptera: Miridae). J. Econ. Entomol. 89, 1053-1059.

Steinkraus, D.C., Tugwell, N.P., 1997. Beauveria bassiana (Deuteromycotina: Moniliales) effects on Lygus lineolaris (Hemiptera: Miridae). J. Econ. Entomol. 32, 79-90.

Thomson, S.V., Hansen, D.R., Flint, K.M., Vandenberg, J.D., 1992. Dissemination of bacteria antagonistic to Erwinia amylovora by honey bees. Plant Dis. 76, 1052-1056.

Yu, H., Sutton, J.C., 1997. Effectiveness of bumble bees and honey bees for delivering inoculum of Giocladium roseum to raspberry flowers to control Botrytis cinerea. Biol. Control 10, 113-122. 